Michael Alex

\title{
Die Einflussnahme der Ministerialbürokratie auf die Entwicklung der Sozialtherapie am Beispiel der Zerschlagung der Sozialtherapeutischen Anstalt Halle/Saale
}

\section{Abstract}

Im März 2014 wurde die Sozialtherapeutische Abteilung der JVA Halle/Saale aufgelöst, nachdem sie schon 2010 ihren Status als selbstständige Einrichtung verloren hatte. An ihre Stelle trat eine kleine Abteilung in der JVA Burg bei Magdeburg mit neuem Konzept und neuen MitarbeiterInnen. Der Beitrag beschreibt die Entwicklung seit der Gründung der SothA Halle im Jahre 2001 und einige der Einflüsse des Justizministeriums Sachsen-Anhalt auf diese Entwicklung.

Schlüsselwörter: Sozialtherapie, strukturelle Mängel, Managementfehler, Justizverwaltung, Wertschätzung

The smash of the social-therapentic institution at Halle/Saale as an example for the influence of the prison administration on the development of social therapy

Abstract

In March 2014 the social-therapentic department (SothA) of the prison at Halle/Saale was closed, having lost the status of an independent institution already in 2010. In its place a small unit was established at the prison of Burg near Magdeburg with a new concept and a new staff. The essay describes the development since the SothA Halle started in 2001 and also some of the influences of the Ministry of Justice in Sachsen-Anbalt on this development.

Key words: Social therapy, structural deficiencies, management problems, prison administration, appreciation

\section{A. Ausgangssituation}

Als Folge des „Gesetzes zur Bekämpfung von Sexualdelikten und anderen gefährlichen Straftaten" vom 26.01.1998 (BGBl. I, 160) war auch Sachsen-Anhalt gezwungen, bis Ende 2002 eine sozialtherapeutische Einrichtung vorzuhalten. Zu diesem Zweck wurde eine bereits geplante Erweiterung auf dem Gelände der Jugendanstalt in Halle im Stadtteil „Frohe Zukunft“ durch einen Neubau mit 140 Haftplätzen umgewidmet, und 
stattdessen eine sozialtherapeutische Anstalt mit 116 Plätzen errichtet. 24 Hafträume wurden als Dienstzimmer für das Personal benötigt. Dank der Initiative der damaligen Justizministerin und ihrer Staatssekretärin sollte die Anstalt entsprechend den Empfehlungen des „Arbeitskreises sozialtherapeutische Anstalten im Justizvollzug e.V.“ ${ }^{\prime}$ personell so ausgestattet werden, dass für 10 Gefangene/Klienten jeweils eine Angehörige oder Angehöriger des Sozialdienstes und des psychologischen Dienstes zuständig sein sollte. Während es gelang, für den psychologischen Fachdienst zehn Fachkräfte mit und ohne Approbation als Psychologische Psychotherapeuten (zuzüglich einer Soziologin für das Qualitätsmanagement) zu einer Versetzung zu motivieren oder neue Kräfte einzustellen, standen für den Sozialdienst schließlich nur sechs Kräfte (teilweise mit abgeschlossener therapeutischer Zusatzausbildung) zur Verfügung, weil die übrigen Planstellen schon anderweitig besetzt waren. Im September 2001 wurden die ersten Gefangenen in die neuerbaute Anstalt verlegt. Das der Einrichtung zugrunde liegende Fachkonzept ${ }^{2}$ war bereits ein Jahr nach Eröffnung der Anstalt nicht mehr umsetzbar, weil frei werdende Stellen im psychologischen Fachdienst nicht wieder besetzt wurden, über einige Stellen im sozialen Fachdienst anderweitig verfügt worden war und die nach dem Fachkonzept als erforderlich anerkannten Stellen im Allgemeinen Vollzugsdienst im Justizministerium nunmehr teilweise für entbehrlich gehalten wurden. „Hilferufe“ von 18 Mitarbeiterinnen und Mitarbeitern, zumeist Angehörige der Fachdienste, und des damaligen Anstaltsleiters im Frühjahr 2002, hielten das Justizministerium nicht davon $\mathrm{ab}$, auch bei reduziertem Personalstand eine Belegung aller 116 Haftplätze zu fordern. Ebenso sah der Hauptpersonalrat noch im Jahre 2004 keine Veranlassung, sich für eine Aufstockung des Personals der SothA einzusetzen, so dass sich Ende 2005 die Situation so darstellte: Von den zehn bei Eröffnung in der SothA Halle tätigen Psychologinnen und Psychologen hatten sechs die Einrichtung inzwischen verlassen, nur ein Teil der Stellen wurde wieder besetzt. Im sozialen Fachdienst waren $4 \frac{1}{2}$ von 10 vorgesehenen Stellen nicht besetzt, im Allgemeinen Vollzugsdienst hatte schon 2002 ein Defizit von 25\% bestanden. In der Antwort auf eine kleine Anfrage im Landtag 3 räumte die Landesregierung Ende 2005 diesen Sachverhalt im Wesentlichen ein, sah aber keinen Änderungsbedarf, da 39 der vorhandenen 116 Haftplätze ohnehin nicht belegt seien. Daran hat sich danach nicht viel geändert. Nach der Stichtagserhebung der KrimZ befanden sich am 31.03.2009 sogar nur 53 Gefangene in der SothA Halle, über all die Jahre war sie die am wenigsten ausgelastete sozialtherapeutische Einrichtung in Deutschland.

Ein besonders auffälliges Beispiel für die Veränderungen der sozialtherapeutischen Praxis infolge der mit dem „Sexualstraftäterbekämpfungsgesetz“ von 1998 eingeleiteten gesetzlichen Neuregelungen ist die Lockerungspraxis. Hatten 1997 noch 52,3\% der Insassen sozialtherapeutischer Einrichtungen selbstständige Vollzugslockerungen, so betrug am 31.03.2003 dieser Anteil nur noch ca. 35\% und sank in den zehn Folgejah-

1 Arbeitskreis, Mindestanforderung, zuletzt veröffentlicht in FS 2007, 100-103.

2 Vgl. Frövel 2000.

3 KA 4/7112.

NK 26. Jg. 4/2014 
ren auf $20 \% .^{4}$ Die SothA Halle zeichnete sich in diesem Zusammenhang durch eine besonders rigide Praxis aus, die die generelle Ablehnung von Vollzugslockerungen im Land Sachsen-Anhalt, das bei allen über Ausgang hinausgehenden Vollzugslockerungen bundesweit seit Jahren das Schlusslicht bildet und auch beim Ausgang nur von Bayern mit noch niedrigeren Zahlen übertroffen wird, ${ }^{5}$ widerspiegelt.

So verwundert es nicht, dass von den ersten 24 Entlassenen aus der SothA Halle kein einziger jemals Urlaub aus der Haft erhalten hat. Drei durften immerhin stundenweise die Anstalt ohne Begleitung durch Bedienstete verlassen, 13 erhielten Ausführungen durch Vollzugsbeamte zu Behörden etc., die übrigen acht kamen erst am Entlassungstag erstmalig wieder vor das Anstaltstor. ${ }^{6}$ Ende 2005 sah es folgendermaßen aus: Von bis dahin insgesamt 58 entlassenen Klienten hatten lediglich 8 (begleitete) Ausgänge und 41 Ausführungen, 9 Klienten kamen erst am Entlassungstag erstmalig aus der Anstalt. Urlauber hatte es in den ersten $4 \frac{1}{2} \mathrm{~J}$ Jahren seit Eröffnung der Anstalt ebenso wenig gegeben wie Verlegungen in den offenen Vollzug. Das hat sich in den folgenden Jahren nur in geringem Umfang geändert. Ein wesentliches Kriterium für die Versagung von Vollzugslockerungen war in den ersten Jahren, wie sich den Vollzugsplänen und Vollzugsplanfortschreibungen jener Zeit durchgängig entnehmen lässt, die „noch nicht erfolgte Aufarbeitung der Straftat“, obwohl im Gesetz nur Missbrauchs- oder Fluchtgefahr als Kriterien für Nichteignung genannt sind. Der Verzicht auf Vollzugslockerungen, obwohl die gesetzlichen Hindernisse kaum jemals anzunehmen waren, war ein zentrales Versäumnis im Behandlungsprozess der SothA Halle in den ersten Jahren, das die konträren Vorstellungen von integrativer Sozialtherapie unter Mitarbeiterinnen und Mitarbeitern mit ihrem jeweils unterschiedlichen Sozialisationshintergrund offenbarte, aber auch ein bezeichnendes Licht auf die Einflussnahme durch das Ministerium der Justiz wirft. Jede über eine Ausführung hinausgehende Öffnung nach außen stand unter dem Zustimmungsvorbehalt des Ministeriums. ${ }^{7}$ Demzufolge musste die Anstalt bei der Planung von begleiteten Ausgängen zunächst die $\mathrm{Zu}$ stimmung des Justizministeriums einholen, nach Durchführung einer vorgegebenen Anzahl von Begleitausgängen dem Ministerium über den Verlauf berichten und um Zustimmung für die nächste Lockerungsstufe (unbegleitete Ausgänge) bitten. Vor der ersten Beurlaubung (Abwesenheit über einen Tag hinaus) musste dieses jeweils etwa sechs Monate in Anspruch nehmende Procedere wiederholt werden, so dass in vielen Fällen seit der ersten Zustimmungsanfrage mittlerweile ein Jahr vergangen war und in der Regel die Entlassung zu einem Zeitpunkt erfolgte, zu dem die Zustimmung zu einer Beurlaubung noch nicht erteilt war.

Ein weiterer die Wahrnehmung der Gefangenen negativ beeinflussender Faktor war im Frühjahr 2002 die Einführung einer nachträglichen Unterbringungsmöglichkeit für Gefangene in Sachsen-Anhalt, die am Ende ihrer Haftzeit aufgrund neuer Erkenntnis-

4 Krimz Stichtagserhebung 2013.

5 Vgl. Greifswalder Inventar für 2007 und davor.

6 Alex 2005, 49.

7 Rundverfügung des Ministeriums der Justiz vom 03.09.1997 zur Gewährung von Vollzugslockerungen, Urlaub sowie Unterbringung im offenen Vollzug - Az.: 4510-303.2-II.1a. 
se für gefährlich gehalten wurden, ${ }^{8}$ eine Regelung, die im Jahre 2004 durch $\$ 66 \mathrm{~b}$ StGB auf ganz Deutschland ausgedehnt wurde. Da viele Klienten der SothA die formellen Voraussetzungen für die nachträgliche Sicherungsverwahrung erfüllten, waren sie urplötzlich mit der Situation konfrontiert, dass im Falle des Ausbleibens von Therapieerfolgen ihre Haft für unbegrenzte Zeit verlängert werden konnte. Da zwischenzeitlich ein entsprechender „Verwahrter“ im Status eines „Untersuchungshäftlings“, weil noch nicht rechtskräftig verurteilt, in die SothA verlegt wurde, ${ }^{9}$ wurde den übrigen Gefangenen die entsprechende Perspektive besonders drastisch vor Augen geführt. ${ }^{10}$

\section{B. Von der selbständigen Anstalt zur sozialtherapentischen Abteilung der JVA Halle}

Bereits im Jahre 2004 hatte der erste Anstaltsleiter resigniert das Handtuch geworfen. Anfang 2008 ließ sich sein Nachfolger in ein anderes Bundesland versetzen und wurde durch den nunmehr dritten Leiter in sieben Jahren ersetzt. Die drei Leitungswechsel gingen jeweils mit einer Neubesetzung der Position der stellvertretenden Anstaltsleitung einher.

Zum 01.03.2010 wurde die Anstalt zu einer Abteilung der Nebenstelle der JVA Halle in der Wilhelm-Busch-Straße umgewidmet, unter anderem mit der Folge, dass ab Anfang 2013 von den bis dahin bestehenden 116 Haftplätzen 32 für den Regelvollzug abgezweigt wurden. Von den nunmehr noch bestehenden 84 Plätzen waren am 31.03.2013 noch 44 mit Klienten der SothA belegt. ${ }^{11}$

Neben der Vermengung mit dem Regelvollzug, die nach kleinen Anfragen im Landtag vom 10.06.2013 ${ }^{12}$ wieder rückgängig gemacht wurde, resultierten aus der Umwandlung von einer selbständigen Anstalt in eine Abteilung weitere in der Literatur aufgeführte Nachteile der Umwandlung von Anstalten in Abteilungen, so insbesondere der Verlust der selbständigen Verfügung über den Personaleinsatz. ${ }^{13}$ Damit war das bewährte Bezugsbetreuer-Modell (Bedienstete aus dem Allgemeinen Vollzugsdienst sind feste Ansprechpartner für eine kleine Gruppe von Klienten) hinfällig geworden.

Im Sommer des Jahres 2012 spitzte sich die Situation zu. Der dritte Leiter, der den Übergang von der selbständigen Anstalt in eine Abteilung der JVA Halle mit vorbereitet hatte, wechselte 2012 in das Justizministerium. Seine Nachfolgerin als fachliche Leitung war zu diesem Zeitpunkt erst ein Jahr in der Anstalt tätig, hatte aber zuvor im Maßregelvollzug gearbeitet.

Die Kritik der Mitarbeiterinnen und Mitarbeiter der SothA an der zusätzlichen Qualitätsminderung der Arbeit nach der Umwandlung von einer selbständigen Anstalt in eine Abteilung der JVA Halle wurde im August 2012 mit der bis zur Auflösung der

8 Straftäter-Unterbringungsgesetz vom 6. März 2002, GVBl. LSA Nr. 12/2002, S. 80).

9 Vgl. BVerfGE 109, 190.

10 Vgl. zu den Problemen Alex StV 26.2. (2006), $105 \mathrm{ff}$.

11 KrimZ, Stichtagserhebung 2013; geringfügig abweichende Zahlen in der Antwort der Landesregierung vom 23.07.2013 auf eine kleine Anfrage vom 10.06.2013, Drucks. 6/2312.

12 KA 6/7956 und KA 6/7957.

13 Arbeitskreis 2007, 100, 102.

NK 26. Jg. 4/2014 
SothA Halle im Frühjahr 2014 fortgesetzten disziplinarischen Abordnung ${ }^{14}$ von zwei „Psychologischen Psychotherapeuten" 15 an andere Justizvollzugsanstalten weitgehend erstickt. Im Juni 2013 wurde auf einer Personalversammlung durch Vertreter des Ministeriums für Justiz und Gleichstellung angedeutet, dass die SothA Halle in die JVA Burg verlegt werden sollte (wo noch immer keine Vollbelegung erreicht war und dem privaten Betreiber ${ }^{16}$ entsprechende Konventionalstrafen gezahlt werden mussten), und angefragt, wer von den Fachdienstmitarbeitern sich eine Versetzung nach Burg vorstellen könne. Die „Bezugsbetreuer“ aus dem AVD wurden gar nicht erst gefragt. Von den 12 Fachdienstmitarbeiterinnen und -mitarbeitern der SothA Halle (8 Psychologen, 4 Sozialpädagogen) haben sich schließlich drei Psychologinnen mit vergleichsweise wenig Erfahrung in der Sozialtherapie nach Burg versetzen lassen, die erfahrensten und teilweise besonders hoch qualifizierten Bediensteten sind ab Anfang des Jahres $2014^{17}$ auf andere Justizvollzugsanstalten im Land verteilt oder anderweitig eingesetzt worden und sind zumeist nicht mehr sozialtherapeutisch tätig. Der Sozialdienst in der neuen sozialtherapeutischen Abteilung in Burg, die zunächst 40 Plätze umfassen soll, ist vollständig mit Kräften ausgestattet, die bisher nicht in der Sozialtherapie und teilweise nicht einmal im Strafvollzug tätig gewesen sind. Verloren gegangen sind damit 13 Jahre Erfahrung in der Arbeit mit dieser schwierigen Klientel und ein mittlerweile weit verzweigtes Netz der Zusammenarbeit mit externen Einrichtungen in Halle zur Optimierung der Entlassungsbedingungen und der Nachsorge.

In einem Schreiben an das Ministerium der Justiz haben Mitarbeiterinnen und Mitarbeiter der SothA Halle im Herbst 2013 deutlich auf die Konsequenzen der Verlegung der Einrichtung hingewiesen, haben dort aber kein Gehör gefunden. Der Rechtsausschuss des Landtages hat sich wiederholt mit dem vom Justizministerium vorgelegten Konzept für die Neuorganisation der Sozialtherapie im Land Sachsen-Anhalt befasst und in der Sitzung vom 14.02.2014 das Vorhaben gebilligt.

\section{Die Evaluation der SothA Halle durch die Universität Halle-Wittenberg}

Die juristische Fakultät der Martin-Luther-Universität war ursprünglich vom Justizministerium des Landes Sachsen-Anhalt mit der Evaluation der Sozialtherapie im Lande beauftragt worden, doch stellte das Land die Finanzierung nach drei Jahren im Jahre 2004 ein, so dass ein neuer Kostenträger gefunden werden musste. Seit August 2005

14 Vgl. dazu Antwort der Landesregierung vom 01.08.2013 auf eine kleine Anfrage vom 10.06.2013, LT-Drucks. 6/2331.

15 Nur 34,5\% der in der Sozialtherapie in Deutschland tätigen Psychologinnen und Psychologen verfügen über eine Approbation als Psychologische Psychotherapeuten, vgl. KrimZ, Stichtagserhebung 2013,88.

16 Die JVA Burg wird nach dem sog. PPP-Konzept (Public-Private-Partnership) betrieben. Privater Betreiber ist die Firma KÖTTER Services.

17 Seit 01.04.2014 ist die sozialtherapeutische Abteilung offiziell der JVA Burg angegliedert, vgl. Vollstreckungsplan vom 01.02.2014. 
wurde das Forschungsvorhaben durch die Deutsche Forschungsgemeinschaft (DFG) gefördert.

In Heft 1/2010 der „Neuen Kriminalpolitik“ haben Seifert und Thyrolf die Ergebnisse einer Befragung von Gefangenen in der Sozialtherapeutischen Anstalt Halle/Saale während der Jahre 2001 bis 2008 dargestellt. Sie kommen zu dem Schluss, dass die Rahmenbedingungen der Sozialtherapie zwar besser seien als die des Normalvollzuges, „aber zwischen Therapiebeginn und Therapieende ist auch eine Bewertungsverschlechterung aus Sicht der Gefangenen zu konstatieren. ${ }^{18}$ Die Gründe hierfür können vielfältig sein, lassen sich aber durchaus konkretisieren. So spiegeln die Daten zumindest teilweise tatsächliche Probleme in der SothA und die Strafvollzugslandschaft des untersuchten Bundeslandes wider, d. h. sie können im Kontext von medial thematisierten Sicherheitsmängeln sowie personellen und organisatorischen Veränderungen interpretiert werden.“ Für die Autorinnen steht fest: „Wenn es nicht gelingt, Lern- und Lebensbedingungen zu schaffen, die zumindest annäherungsweise realitätsnah sind, die wohlwollend unterstützt werden und in einem angstfreien Raum erfolgen, werden diese kostenintensiven Resozialisierungsprogramme kaum einen Beitrag zu einer merklichen Reduzierung der Rückfallrate leisten." ${ }^{19}$

Hinzu kommt die von Seifert/Thyrolf angesprochene Kluft zwischen den Erfahrungen der Gefangenen aus der Therapie und ihren Erlebnissen im Anstaltsalltag, wo beispielsweise ein freundlicher Umgang untereinander oder das Ausreden lassen des Gegenübers vermisst werden. Da in der sozialtherapeutischen Anstalt auch „altgediente“ Beamte, die sozialtherapeutischen Interventionen ablehnend gegenüber stehen, beschäftigt sind und auch gegen den expliziten Willen sozialtherapeutisch orientierter Mitarbeiterinnen und Mitarbeiter ihre Tätigkeit mit maßgeblichen Befugnissen noch immer unbeirrt fortsetzen können, prallten Gegensätze aufeinander, die den Gefangenen nicht verborgen bleiben konnten und ihren Alltag bestimmten. ${ }^{20}$ Doch noch im Juni 2006 hatte das Justizministerium des Landes Sachsen-Anhalt zu dieser Problematik nicht mehr zu sagen, als dass der Anstaltsleiter seit seinem Amtsantritt die Kommunikations- und Organisationsstrukturen der Einrichtung verändert habe, um eine Integration von Behandlungs- und Sicherheitsaspekten zu fördern. Anlass für eine Intervention wurde nicht gesehen, mit der Zeit seien positive Entwicklungen zu erwarten. ${ }^{21}$ Auch die Intervention einer Gruppe von Mitarbeiterinnen und Mitarbeitern der SothA Halle beim Anstaltsbeirat Ende des Jahres 2006 wegen Defiziten im Führungsverhalten und der Nicht-Anerkennung von therapeutischer Arbeit blieb unbeachtet.

Seifert/Thyrolf zitieren zahlreiche Veröffentlichungen, in denen das therapeutische/ soziale Klima als ein wesentlicher Beitrag für rückfallvermindernde Sozialtherapie herausgestellt worden ist. ${ }^{22}$ Immer wieder ist dort davon die Rede, dass die Gefangenen die Bedingungen im Vollzug „annehmen“ müssen, damit sie sich den angebotenen

18 Seifert/Tyrolf NK 22. 1. (2010), 23, 30.

19 Dies. 30.

$20 \mathrm{Zu}$ den Anforderungen an das Personal vgl. Wischka 2004, 340ff.

21 Antwortschreiben des JM-LSA vom 23.06.2006 an den Verfasser.

22 Seifert/Thyrolf NK 22.1. (2010), 23,29.

NK 26. Jg. 4/2014 
Maßnahmen öffnen. „Gefangene werden eher ihre Einstellungen überdenken und an ihrem Verhalten arbeiten, also erreichbar sein, wenn sie Resozialisierung in einem offenen, positiven und von Mitbestimmung geprägten Klima erleben. (...) Vor allem pädagogische und therapeutische Maßnahmen werden umso effektiver sein, je mehr sie in die Gesamtstruktur der Anstalt eingebunden sind, denn die Behandlung endet nicht an der Tür des Gruppentherapieraums“. ${ }^{23}$ Zentraler Aspekt bei der Herstellung eines therapeutischen Klimas ist darüber hinaus die Kontinuität der Beziehungen, das interaktive Miteinander. ${ }^{24}$ Die Koordination des therapeutischen Vorgehens aller an der Behandlung Beteiligten muss deshalb auch beinhalten, dass Teams über längere Zeit zusammenarbeiten und nicht in schneller Folge ausgetauscht werden. ${ }^{25}$ Aus unterschiedlichen Gründen ist in der Sozialtherapeutischen Anstalt Halle diesen für eine erfolgreiche Rückfallverhinderung essentiellen Voraussetzungen nur wenig Beachtung geschenkt worden, so dass es zu einer hohen Fluktuation beim Personal gekommen ist, erhebliche Spannungen innerhalb des Personals in Kauf genommen wurden und der Sicherungsaspekt den Resozialisierungsansatz in den Hintergrund schob. Die von Seifert/Thyrolf dargestellten Ergebnisse der Gefangenenbefragung ließen in Verbindung mit den objektiven Defiziten in der Struktur des sozialtherapeutischen Prozesses befürchten, dass die SothA Halle den Erwartungen an eine erfolgreiche Sozialtherapie weniger gut gerecht werden würde, als dies bei optimaler Nutzung der vorhandenen Ressourcen möglich gewesen wäre. Negative Folgen dieser Entwicklung für die Rückfallvermeidung werden aber zu Lasten des gesamten sozialtherapeutischen Ansatzes in Deutschland gehen, obwohl sie „hausgemacht" sind.

Im abschließenden Arbeitsbericht zum von der Deutschen Forschungsgemeinschaft geförderten Projekt „Kriminologische Evaluation der Sozialtherapeutischen Anstalt Halle (Saale)" vom Oktober 2013 (noch nicht veröffentlicht) fassen Bussmann und Richter die Gesamtergebnisse der Evaluation u. a. wie folgt zusammen:26

„(..) Eine moderate Veränderung einiger dynamischer Risikofaktoren ist messbar, so ist z.B. die Förderung von psychischer Gesundheit, Realitätsnähe und Verantwortungsübernahme gelungen, die Bearbeitung zwanghafter Tendenz und der Suchtproblematik jedoch nicht (erfolgt).

Größte Schwierigkeit während der Therapie stellt das „nicht ausprobieren können“ der gelernten Inhalte unter „realistischen Bedingungen“ dar. Die Probanden erhalten kaum Vollzugslockerungen und starten erst nach der Haftentlassung mit der praktischen Umsetzung des Gelernten. Eine professionelle engmaschige Nachbetreuung, in der die therapeutischen Bemühungen fortgesetzt werden, gibt es für die meisten Entlassenen nicht, auch wenn mit der Einrichtung der „Forensischen Ambulanz des Landes Sachsen-Anhalt“ Bemühungen in diese Richtung sichtbar sind.

23 Dies. 29.

24 Vgl. Rehn NK 15.2. (2003), 66, 68.

25 Wischka 2001, 127.

26 Bussmann/Richter 2013, $20 f$. 
Insgesamt lassen sich bei der Betrachtung eines durchschnittlich 5,5-jährigen Katamnese-Zeitraumes bezüglich der registrierten Rückfälligkeit keine entscheidenden Vorteile für die Probanden der Sozialtherapie gegenüber den Entlassenen anderer sachsenanhaltinischer Haftanstalten ausmachen. Erneute Sanktionen wurden für $42,4 \%$ der Untersuchungsgruppe und für 46,5\% der Vergleichsgruppe ausgesprochen. Eine Sexualstraftat nach der Entlassung wurde für 6,7\% der Probanden aus beiden Gruppen registriert. Abbrecher der Sozialtherapie weisen (mit Ausnahme von Verkehrsdelikten) keine erhöhte Kriminalität auf. Die Rückfallgeschwindigkeit ist für die Teilnehmer der Sozialtherapie mit ca. 19 Monaten geringer als für die Vergleichsgruppe mit ca. 14 Monaten).

Die kleine Gruppe der mit einem Sexualdelikt rückfällig gewordenen Personen weist eine stärkere Ausprägung statischer und dynamischer Risikofaktoren auf und konnte von dem Angebot der SothA in geringerem Maße profitieren. Nach ihrer Entlassung zeigen diese Probanden größere Probleme bei der Integration in ein Berufsleben, finanziellen Angelegenheiten und bzgl. der eigenen Gesundheit. Außerdem sind sie weniger in vorhandenen Nachsorgeangeboten eingebettet.

Aktuell wird die SothA als Abteilung einer anderen Justizvollzugsanstalt betrieben und sieht sich im Rahmen der Strukturreform des Justizvollzuges Sachsen-Anhalts vor weiteren Veränderungen. So bedarf es zwingend einer konzeptionellen Überarbeitung, wie zukünftig sozialtherapeutische Arbeit in Sachsen-Anhalt gestaltet werden soll, wobei wissenschaftlich erarbeitete Indikationskriterien und empirisch bestätigte Wirkprinzipien wegweisend sein sollten.

Es bleibt zu hoffen, dass damit ein Ausbau des sozialtherapeutischen Angebotes und der Weiterqualifizierung des Personals sowie eine Verbesserung der Entlassungsvorbereitungen insbesondere eine engere Zusammenarbeit mit den nachsorgenden Einrichtungen einhergeht. Die Abkehr von der Fokussierung auf eine bestimmte Tätergruppe hin zu einer prognostischen Auswahl der Teilnehmer, so dass nicht mehr das Anlassdelikt, sondern eine bestehende erhebliche Gefährlichkeit des Täters die Grundlage der therapeutischen Zuwendung bildet, stellt außerdem die Weichen für einen effektiveren Einsatz der knappen Behandlungsressourcen.“27

\section{Fazit}

Das negative Ergebnis der kriminologischen Evaluation dürfte die Entscheidung des Justizministeriums, die SothA in Halle zu zerschlagen, ebenso befördert haben wie die im Sommer 2012 offen zu Tage getretenen Spannungen im Fachteam. Auf diese Weise konnten die vorrangig fiskalischen Überlegungen für eine Verlagerung der Sozialtherapie in die JVA Burg hinter fachlichen Erwägungen versteckt und auch der Landtag von der Sinnhaftigkeit des neuen Konzepts überzeugt werden. Dabei war gerade die Lan-

27 Vgl. insoweit und zu den Problemen bei der Evaluation der Sozialtherapie in Sachsen Wößner/Schulz 2013, $130 \mathrm{ff}$. und grundsätzlich zu den Problemen der Sozialtherapie im Land Sachsen-Anhalt unter den gegenwärtigen gesellschaftlichen Bedingungen Seifert 2014.

NK 26. Jg. 4/2014 
desregierung hauptsächlich verantwortlich für wesentliche Mängel, die bei der Evaluation festgestellt wurden. Die Ausgangslage bei Eröffnung der Sozialtherapeutischen Anstalt Halle im Jahre 2001 war nicht ideal. Gegen den Widerstand einer örtlichen Bürgerinitiative $^{28}$ wurde ein ursprünglich für den Regelvollzug geplantes Gebäude auf dem Gelände der damaligen Jugendanstalt in eine sozialtherapeutische Anstalt mit 116 Plätzen umgewidmet, in großem Umfang Fachpersonal mit sehr unterschiedlichem Erfahrungshintergrund angeworben und auch im allgemeinen Vollzugsdienst eine Versetzungspraxis ausgeübt, die die Heterogenität erhöhte. Nicht ohne Grund gehen die Empfehlungen des Arbeitskreises Sozialtherapeutische Anstalten im Justizvollzug e.V. an die räumlichen Voraussetzungen von einer maximalen Größe von 60 Haftplätzen aus. ${ }^{29}$ Der Auftrag an die SothA Halle lautete ausdrücklich, sich vorrangig der Behandlung von Sexualstraftätern zu widmen. Demgemäß betrug der Anteil der Sexualstraftäter in den ersten vier Jahren durchweg mehr als 84\%. Erst seit 2008 sank er auf unter $80 \%$ auf durchschnittlich $70 \%$ mit einem Niedrigstanteil von $55 \%$ am 31.03 .2013 , dem Jahr vor der Schließung. ${ }^{30} \mathrm{Zu}$ diesem Zeitpunkt war die Datenerhebung durch das Evaluationsteam seit drei Jahren abgeschlossen, so dass Aussagen über Auswirkungen veränderter Aufnahmekriterien nicht mehr möglich sind. Der Abschlussbericht legt allerdings nahe, dass mit zunehmender Erfahrung dem Rückfallrisiko als Aufnahmekriterium mehr Gewicht beigemessen wurde als bei Eröffnung der SothA Halle. Doch selbst diese Problematik war eher den Vorgaben der Landesregierung geschuldet. Dem Anliegen des ersten Anstaltsleiters, die Teilnahme der Klienten an den therapeutischen Maßnahmen analog $\$ 37$ StVollzG als Weiterbildungsmaßnahme zu vergüten, wie das seinerzeit in mehreren Bundesländern gängige Praxis war und seit dem 01.06.2013 für Sicherungsverwahrte bundesweit gilt, ${ }^{31}$ widersprach im Jahre 2001 das Finanzministerium. Sofern Klienten der SothA Halle einer (Halbtags-) Arbeit neben der Teilnahme an therapeutischen Angeboten nachgingen, war die Vergütung in der Regel deshalb so niedrig, dass das Niveau des Taschengeldes gemäß $\$ 199$ StVollzG nicht erreicht wurde. Ein Wechsel in die Sozialtherapie war für viele Gefangene deshalb schon aus finanziellen Gesichtspunkten wenig attraktiv, weil er mit dem Verlust des bisherigen Arbeitsplatzes und der Vergütung für eine Vollzeittätigkeit in der Stammanstalt verknüpft war.

Ebenso war die angespannte Personalsituation auf die fehlende Bereitschaft der Landesregierung, frei werdende Fachdienststellen wieder zu besetzen, sowie das Bestreben, die Sozialtherapeutische Anstalt als „Abstellgleis“ für in anderen Anstalten nicht mehr gewünschte Angehörige des Allgemeinen Vollzugsdienstes, denen jegliches Verständnis für sozialtherapeutische Belange fehlte, zu nutzen, zurückzuführen. Hinzu kommt, dass in den 13 Jahren des Bestehens der SothA Halle vier Leiter bzw. Leiterinnen für die Geschicke der Einrichtung verantwortlich waren, die teilweise ihre Tätigkeit lediglich als Schritt auf der Karriereleiter betrachteten und unterschiedlichste kon-

28 Vgl. dazu Alex NK 13.4. (2001), 4 f.

29 Arbeitskreis, Mindestanforderung 2007, 102.

30 KrimZ, Stichtagserhebung 2013.

31 Vgl. etwa $\$ 37$ SVVollzG LSA. 
zeptionelle Veränderungen auf Grundlage ihrer Persönlichkeit und ihrer Vorerfahrungen durchzusetzen versuchten.

Als größte Schwierigkeit während der Therapie wird in der Evaluation die fehlende Möglichkeit, das in der Therapie Erlernte unter realistischen Bedingungen zu erproben, genannt. Die Geringschätzung von Vollzugslockerungen für eine angemessene Entlassungsvorbereitung war ein Punkt, auf den früh aufmerksam gemacht worden war, ${ }^{32}$ ohne dass das Ministerium der Justiz bereit war, von der rigiden Lockerungspraxis im Land Sachsen-Anhalt abzurücken. Der Antwort der Landesregierung vom 23.07.2013 auf eine der Kleinen Anfragen vom 10.06.2013 ist u.a. zu entnehmen, dass von den 48 Gefangenen in der SothA am 03.07.2013 gerade einmal zwei (!) im offenen Vollzug betreut wurden. ${ }^{33}$

Nur am Rande wird im Abschlussbericht der kriminologischen Evaluation erwähnt, dass die SothA in den letzten vier Jahren ihres Bestehens als Abteilung einer anderen Justizvollzugsanstalt betrieben wurde, obwohl diese Umwidmung wahrscheinlich der bedeutsamste Schritt zur Zerschlagung der Einrichtung war. Nach $₫ 123$ StVollzG sind grundsätzlich von den übrigen Vollzugsanstalten getrennte selbstständige sozialtherapeutische Anstalten für den Vollzug der Sozialtherapie vorzusehen. Nur aus besonderen Gründen sollte die Einrichtung von Abteilungen in anderen Vollzugsanstalten möglich sein, weil der Gesetzgeber eine Zurückdrängung der sozialtherapeutischen Ansätze durch den Regelvollzug befürchtete. Das Regel-Ausnahme-Verhältnis ist mittlerweile aus Kostengründen in der Praxis umgedreht worden. Am 31.03.2013 gab es in Deutschland nach der Stichtagserhebung der Kriminologischen Zentralstelle noch 6 selbstständige sozialtherapeutische Anstalten, aber 67 sozialtherapeutische Abteilungen. Die Entwicklung der SothA Halle seit der Integration in die JVA Halle zeigt die fatalen Konsequenzen für die Sozialtherapie. Sozialtherapeutische Abteilungen können nur funktionieren, wenn die Anstaltsleitung die sozialtherapeutischen Bemühungen respektiert und fördert. Eingriffe in den Personalbestand oder gar die Vermischung mit dem Regelvollzug und den dort bestehenden subkulturellen Strukturen sind auf Dauer tödlich für Sozialtherapie.

Wenn es der Aufsichtsbehörde gelingt, die Verantwortung für Defizite im therapeutischen Prozess unter Vernachlässigung der eigenen Versäumnisse allein der sozialtherapeutischen Einrichtung aufzubürden, ist es ein Leichtes, der Öffentlichkeit die Schließung der Einrichtung plausibel zu machen und vorzugaukeln, mit dem Neubeginn werde alles besser und auf Synergieeffekte zu verweisen, weil nun auch die Insassen einer Hochsicherheitsanstalt in den Genuss von Sozialtherapie kommen können. Insoweit hat die Landesregierung viel Fingerspitzengefühl bewiesen.

Doch hätten sämtliche von dem Forschungsteam gemachten Vorschläge zur künftigen Konzeption des Strafvollzuges Sachsen-Anhalt bezüglich der Sozialtherapie auf- 
bauend auf den gewachsenen Strukturen ohne weiteres im Rahmen der SothA Halle umgesetzt werden können. Empfohlen wurden: ${ }^{34}$

- Einführung eines strukturierten, landeseinheitlichen Auswahlverfahrens

- Ausweitung des sozialtherapeutischen Angebots und stärkere Berücksichtigung Sozialer Arbeit

- Ausbau des „therapeutischen Milieus“

- Möglichkeit der Gewährung von Lockerungen durch die Anstalt

- Einführung eines Übergangsmanagements

- Ausbau der externen Relapse Prevention

Sicher war das Team der SothA mit Mitarbeitern mit vielen Vorerfahrungen ebenso wie Neulingen im Strafvollzug, mit Angehörigen des Allgemeinen Vollzugsdienstes, die dem DDR-Strafvollzug nachtrauerten, und anderen, die sich bewusst für die Sozialtherapie und gegen den Regelvollzug entschieden hatten, um intensiver mit Gefangenen arbeiten zu können, für die jeweilige Anstaltsleitung nicht immer leicht zu steuern und für ein streng hierarchisch strukturiertes Ministerium von Anfang an nicht einschätzbar, andererseits war die Gründungsphase der SothA von für den Öffentlichen Dienst außergewöhnlicher Kreativität und großem Engagement geprägt. Statt dieses Potential zu nutzen, neigte das Justizministerium und - nach der Umwandlung in eine Abteilung der JVA Halle - auch die Anstaltsleitung dazu, den Widerspruch gegen behandlungsfeindliche Entwicklungen zu unterdrücken, und war damit erfolgreich. Dennoch ist es den Mitarbeiterinnen und Mitarbeitern der SothA Halle in den 13 Jahren des Bestehens der Einrichtung gelungen, eine Vielzahl von Angehörigen des allgemeinen Vollzugsdienstes für die Idee der Sozialtherapie zu begeistern und als Bezugsbetreuerinnen und Bezugsbetreuer oder in anderer Weise in die sozialtherapeutischen Prozesse einzubinden. Hinzu kommt die in 13 Jahren erreichte Vernetzung mit Institutionen und Personen außerhalb des Vollzuges. All diese Leistungen zur Verbesserung der Resozialisierungsbedingungen sind dem Auflösungsinteresse des Justizministeriums geopfert worden.

Ob mit dem „Neuanfang“ in der JVA Burg der verheißene „Schub“ für die Sozialtherapie in Sachsen-Anhalt eintreten wird, darf getrost bezweifelt werden. Die auf „freiem Feld“ errichtete Anstalt ist noch abgeschotteter als die Einrichtung in Halle, die Verkehrsanbindung mehr als dürftig und die Lockerungspraxis nicht weniger rigide als in Halle. Insofern ist zu befürchten, dass die Resozialisierungserfolge nicht größer sein werden als in Halle, dass aber auf einen großen Erfahrungsschatz und Fortbildungserfolge sowie die Vernetzung, die in 13 Jahren in Halle erreicht worden ist, verzichtet wird, um „Ruhe“ zu haben. Das macht den Stellenwert, den Sozialtherapie im Denken der Justizverwaltung einnimmt, deutlich. Letztlich geht es nur darum, den gesetzlichen Vorgaben Genüge zu tun, ohne wirklich die Resozialisierungsbemühungen um vermeintlich hoch gefährliche Straftäter zu verstärken. Das einzige Risiko wird in Zwischenfällen während der Inhaftierung gesehen, das Verhalten nach der Haftentlassung ist relativ gleichgültig. Die Verschlechterungen des sozialen Klimas in der SothA

34 Bussmann/Richter 2013, 18.

THEMEN 
Halle waren somit keineswegs auf zufällige Ereignisse zurückzuführen, sondern sind ebenso wie der frühzeitige Verzicht auf die weitere Finanzierung der Begleitforschung - gezielt in Kauf genommen worden, obwohl man sich der Risiken für den Resozialisierungsprozess bewusst war.

\section{Literatur:}

Alex Sozialtherapie als Alibi?, in: NK 13.4. (2001), 4-5

Alex (2005) Aktuelle Entwicklungen der Sozialtherapie in Deutschland, in: Strafverteidigervereinigungen (Hrsg.), Opferschutz, Richterrecht, Strafprozessreform, 43-53

Alex Sozialtherapie unter den Bedingungen der Gesetzesverschärfungen seit 1998 unter besonderer Berücksichtigung von vorbehaltener und nachträglicher Sicherungsverwahrung,in: Strafverteidiger 26.2. (2006), 105-108

Arbeitskreis Sozialtherapentische Anstalten im Justizvollzug e.V. Sozialtherapeutische Anstalten und Abteilungen im Justizvollzug. Mindestanforderung an Organisation und Ausstattung. Indikationen zur Verlegung, FS 56.3. (2007), 100-103

Bussmann/Richter (2013) Arbeitsbericht zum von der Deutschen Forschungsgemeinschaft geförderten Projekt: Kriminologische Evaluation der Sozialtherapeutischen Anstalt Halle (Saale). Unveröffentlichtes Manuskript.

Frövel (2000) SothA LSA. Fachkonzept. Unveröffentlichtes Manuskript

Greifswalder Inventar zum Strafvollzug, Lehrstuhl für Kriminologie, abrufbar unter: http://www.uni-greifswald.de/ 1s3/gis.html

Kriminologische Zentralstelle (KrimZ) Sozialtherapie im Strafvollzug. Stichtagserhebung jeweils zum 31.03. eines Jahres. Wiesbaden (abrufbar unter: www.krimz.de)

Rehn Sozialtherapie - Anspruch und Wirklichkeit 2003, in: NK 15.2. (2003), 66-69

Seifert (2014) Der Umgang mit Sexualstraftätern. Bearbeitung eines sozialen Problems im Strafvollzug und Reflexion gesellschaftlicher Erwartungen

Seifert/Thyrolf Das Klima im Strafvollzug. Eine Befragung von Gefangenen einer sozialtherapeutischen Einrichtung, in: NK 22.1. (2010), 23-31

Wischka (2001) Die Faktoren Milieu, Beziehung und Konsequenz in der stationären Therapie von Gewalttätern, in: Rehn, Wischka, Lösel, Walter (Hrsg.), Behandlung „gefährlicher Straftäter“, 125-149

Wischka (2004) Wohngruppenvollzug, in: Pecher (Hrsg.) Justizvollzugspsychologie in Schlüsselbegriffen, 335-347

Wößner/Schulz (2013) Sozialtherapeutisch behandelte Sexual- und Gewaltstraftäter: Erste Ergebnisse, in: Wößner, Hefendehl, Albrecht (Hrsg.), Sexuelle Gewalt und Sozialtherapie, $107-140$ 
Kontakt:

Michael Alex,

Freier Mitarbeiter,

Rubr-Universität Bochum,

Lebrstubl für Kriminologie, Kriminalpolitik, Polizeiwissenschaft

Universitätsstr. 150,

44801 Bochum,

michel.alex@rub.de

\section{Strafrecht und Psychiatrie}

Der »Fall Mollath « und die Folgen

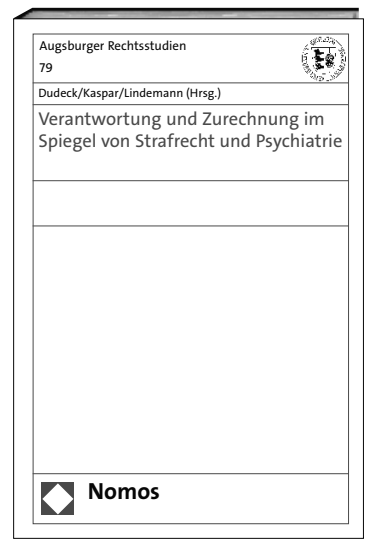

Verantwortung und Zurechnung im Spiegel von Strafrecht und Psychiatrie

Herausgegeben von Prof. Dr. med. Manuela

Dudeck, Prof. Dr. Johannes Kaspar und

Prof. Dr. Michael Lindemann

2014, 232 S., geb., 59,-€

ISBN 978-3-8487-1178-9

(Augsburger Rechtsstudien, Bd. 79)

www.nomos-shop.de/22452

Strafjustiz und Psychiatrie widmen sich, wenn auch aus unterschiedlicher Perspektive, dem Umgang mit „abweichendem Verhalten“. Die dabei auftretenden rechtlichen, medizinischen und ethischen Probleme, die sich nicht zuletzt im Fall „Mollath" gezeigt haben, werden im vorliegenden Band von Expertinnen und Experten aus beiden Disziplinen beleuchtet.

Bestellen Sie jetzt telefonisch unter 07221/2104-37.

Portofreie Buch-Bestellungen unter www.nomos-shop.de

Alle Preise inkl. Mehrwertsteuer

Nomos 